\title{
Meandering Fractals in Water Resources Management
}

\author{
Levent Yilmaz \\ Nisantasi University Neocampus, Maslak, Istanbul, Turkey
}

Email address:

levent.yilmaz@nisantasi.edu.tr

\section{To cite this article:}

Levent Yilmaz. Meandering Fractals in Water Resources Management. Applied and Computational Mathematics. Vol. 9, No. 2, 2020, pp. 26-29. doi: 10.11648/j.acm.20200902.12

Received: April 29, 2019; Accepted: May 21, 2019; Published: May 18, 2020

\begin{abstract}
Fractal dimension is a measure for the degree of complexity or that of fractals. An alternative to fractal dimension is ht-index, which quantifies complexity in a unique way. Back to your question, the physical meaning of fractal dimension is that many natural and social phenomena are nonlinear rather than linear, and are fractal rather than Euclidean. We need a new paradigm for studying our surrounding phenomena, Not Newtonian physics for simple systems, but complexity theory for complex systems, Not linear mathematics such as calculus, Gaussian statistics, and Euclidean geometry, but online mathematics including fractal geometry, chaos theory, and complexity science in general. A channel is characterized by its width, depth, and slope. The regime theory relates these characteristics to the water and sediment discharge transported bye the channel empirically. Empirical measurements are taken on channels and attempts are made to fit empirical equations to the observed data. The channel characteristics are related primarily to the discharge but allowance is also made for variations in other variables, such as sediment size.
\end{abstract}

Keywords: Natural Dimensions, Nonlinearity, Fractals, Meanders

\section{Introduction}

Bank full discharge is generally considered to be the dominant steady flow which would generate the same regime channel shape and dimensions as the natural sequences of flows would. This is because investigation on the magnitude and frequency of sediment transport have determined that for stable rivers the flow which in the longer term transports most material has the same frequency of occurrence as bankfull flow. For stable gravel-bed rivers, this is considered to be the 1. 5-year flood.

The objective of regime theory is to predict the size, shape, and slope of a stable alluvial channel under given conditions. A channel is characterized by its width, depth, and slope. The regime theory relates these characteristics to the water and sediment discharge transported bye the channel empirically. Empirical measurements are taken on channels and attempts are made to fit empirical equations to the observed data. The channel characteristics are related primarily to the discharge but allowance is also made for variations in other variables, such as sediment size.

For practical purposes, rivers are preserved to be in equilibrium (in regime) or in quasi-equilibrium of this characteristics have not changed over a long period of time. Canals usually maintain constant discharge and regime relations may, therefore, be established using field data. However, field measurements for rivers are not usually suitable for establishing laws for rivers in regime.

If you use a ruler of $\mathrm{k}=1000$ meteres you will need $\mathrm{K}$ rulers to run entire river meander curvature. If you use a ruler of $1=500$ meters you need L rulers and sucessivily. What is the physical meaning of fractal dimension?

The numbers of rulers necessary to measure a meander curvature line $\mathrm{M}$ is proportional the length of ruler $\mathrm{m}$ with a exponent $\mathrm{D}$, where here $\mathrm{D}$ is a constant that define the dependence between the number of rules and the length of ruler and is knowed as fractal dimension for measure a river meander curvature.

It is intended to calculate fractal dimension slightly undulating line. It is found one code from net on boxcounting method [3] and used for slightly undulating surface that is not given correct answer. I also have $\mathrm{x}$ and $\mathrm{z}$ value of corresponding line. Is it possible to calculate from these value by any software/ code [3], A characteristic feature of fractals is their fine structure. An object is known to have fine structure if it has irregularities at arbitrarily small scale. 'Fractal dimension' attempts to quantify the fine structure by 
measuring the rate at which increased detail becomes apparent as we examine a fractal ever more closely. Fractal dimension indicates the complexity of the fractal and of the amount of space it occupies when viewed at high resolution. All definitions of dimension depend on measuring fractals in some way at increasingly fine scales.

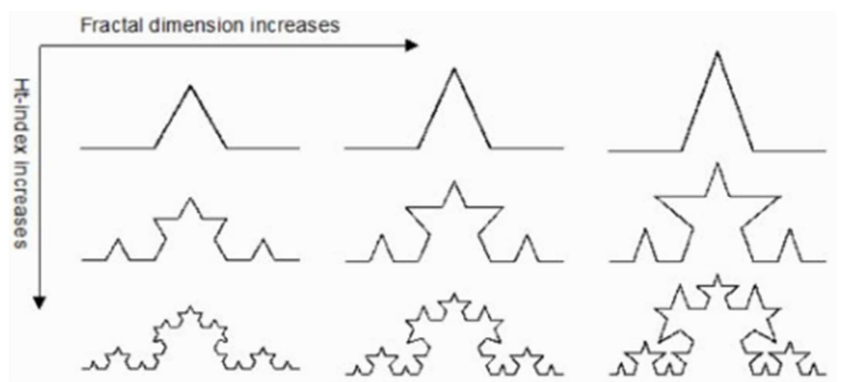

Figure 1. Both the ht- index and fractal dimensions, characterizing fractals from different perspectives [1].

A Fractal, strictly speaking, has no 'physical meaning". It is like asking about some curve we see on some Cartesian 2D coordinate frame "what is its physical meaning? Or the curve, the function which we may have available to help us understand it and the frame of reference are all constructs of what we can now say for purposes of brevity is our intuition and our urge to express ourselves in ways that somehow help us deal with or cope with actions we have to take either now or in the future.

Thus the lines we see on the graph paper have no physical meaning, perse. But that does not mean they have no 'use' In fact "use" is perhaps the best notion of "meaning". Their use if those who may be ablet to co further with those constructs and incorporate them into models they might work with in regard to various inquiries in scinece. Unfortunatelly, there has been litte inquiry into just how and in which way and why fractals may be of use We only tend to 'look at the computer screens" and think that we 'are seeing " something beyond some interesting calculations in complex number space.

In the end, complex numbers and their spaces are of far more use than real numbers and Euclidean style geometries. Hopefully we will be able to hone our intuitions to make use of them and of fractals in a wide range of pursuits and, among them, would be those understandings of ourselves and matters of human engagement which cannot begin to be approached with real number spaces and Eu6clidean assumptions about "reality".

"Reality" itself is an entirely flawed concept which is rooted in our intuitions and imaginations being locked into a limited 'real number/Euclidean/ Cartesian' model of thinking and expressing ourselves. When we then speak of 'reality." we are expressly bringing up the intrincis nonsense and pardox of Cartesian coordinates and the real numbers. Fractals are the first message or signal to us that we can, in the long run, learn more about the universe and about ourselves via the creative "use; of complex numbers and indeed of complex number spaces and those number spaces further down the road of honing of intuitions such as quaternions and octonions as well. Their beauty is a great lure and clue that there is much more than meets the eyes in our numbers and that complex numbers can enable us and our mind's eye to see what real numbers cannot [6].

That is then the first step to using them and using fractal awarenss within our other engagements with the so called 'physical world".

\section{Method}

Most of the objects found in nature possess irregular shapes that can notbe quantified with the help of standard Euclidian geometry. In many cases these objects have a peculiar character of self similarity where the part of the object looks like the whole [1]. Such objects are known as fractals and the associated degree of complexity of shape, structure and texture is quantified interms of Fractal dimension (Figure 2). Natural fractals do exhibit self similarity and scale invariance, however this is present to a limited extent [2], For example part of a cauliflower may look like the whole, if a further division is made the resulting part may not resemble much with the original cauliflower after several steps. The concept of fractal was first introduced by Mandelbrot in the year 1980 [3] he showed that the concept of fractal can be used to quantify the complexity of shape associated with irregular geometry.
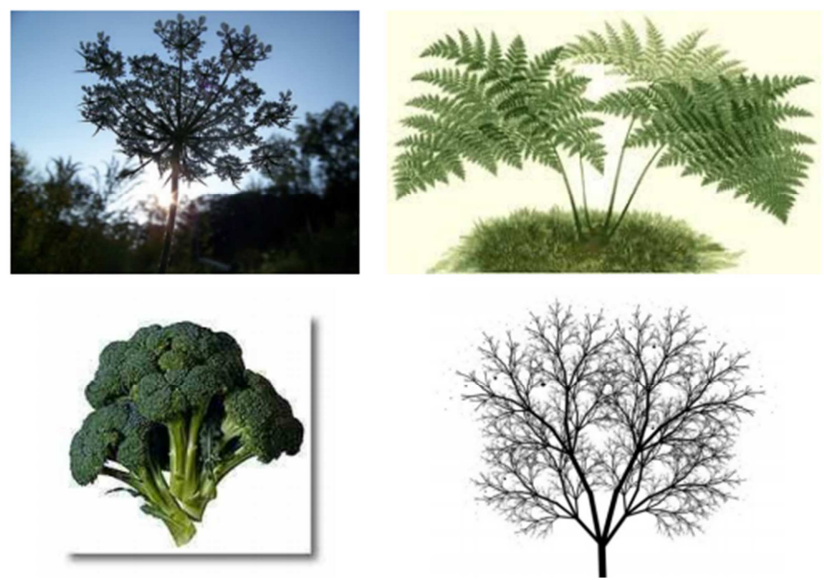

Figure 2. Examples of naturally occurring fractal patterns in natüre [2].

\subsection{River Meander Curvature Fractals}

Fractal dimension of the curve is found from the slope of the best fitting straight line to the data as (Fractal dimension $=1-\mathrm{m}$ ), where $\mathrm{m}$ is the slope of the straight lime.

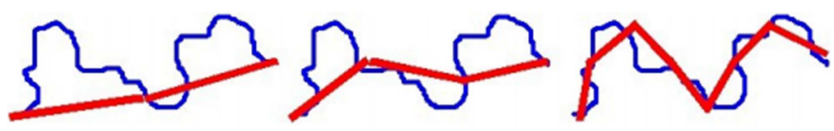

Figure 3. Irregular shape of a line is analysed using ruler method.

Richardson's plot technique using rulers or segments of different sizes [4].

It is seen from Figure 3 that for a given line with irregular shape, the number of segments or rulers of a given size increases as the size of the ruler is decreased. This results in 
different measures of the length of the curved line the complexity of the shape is related to this difference. For a straight line the measurements made using different sizes of rulers or line segments result in the same length where as for complex curves the measured distance is larger and larger as smaller and smaller ruler sizes are used. The fractal dimension is related to the complexity of shape associated with the curve and a higher fractal dimension stands for a higher degree of complexity of the pattern analysed.

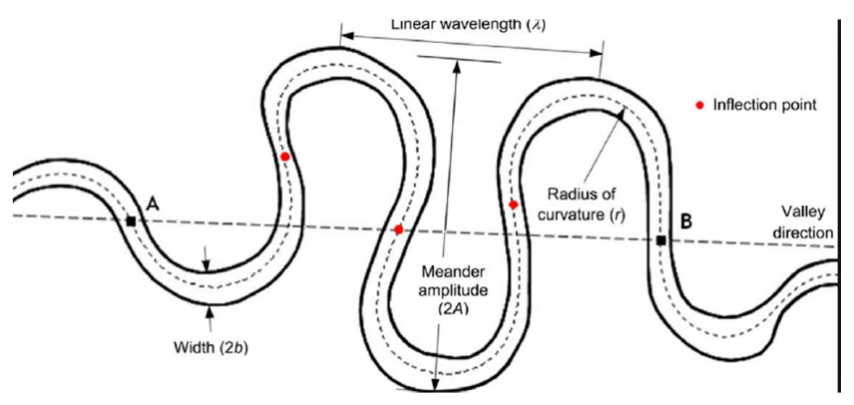

Figure 4. River meander curvature fractals [7].

If the object can be represented by a two-dimensional binary images in a computer screen or a matrix, which can be input from a digital camera or a image scanner the fractal dimension estimation can be described as follows.

For an object in two-dimensional Euclidean space, the mass-radius (MR) relation is expressed as the mass included is proportional to the square of the circle of radius $r$ or:

$$
\mathrm{M}(\mathrm{r})=r^{2}
$$

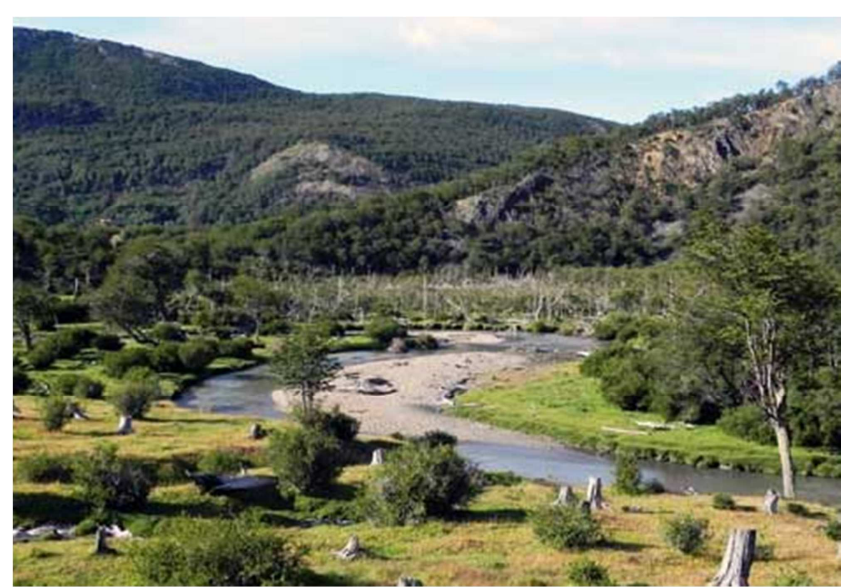

Figure 5. Meander Curvature in Nature as defining the Fractal Dimensions [7].

As an example, the area of a square measured by using circular discs of increasing sizes is directly proportional to the square of the radius of the disc used for the measurement. The power law exponent " 2 " is therefore the Euclidean dimension (a square is two-dimensional); however, the mass of a fractal object changes with a fractional exponent such that $(1<\mathrm{D}<2)$ as:

$$
\mathrm{D} M(\mathrm{r})=\mathrm{r}
$$

From this power law the fractal dimension " $\mathrm{D}$ " of the object can be found as $\log (\mathrm{r})$.

$$
\log (\mathrm{M}(\mathrm{r}))=\mathrm{D}
$$

Here D is the slope of the straight line describing the log (M (r)) versus $\log (\mathrm{r})$. the two-point correlation function (C (r)) is related to the MR relation which can be used to determine the fractal dimension. For a fractal, $\mathrm{C}$ (r) decays as per the power law of a measuring distance (ruler size) $r$ :

$$
\mathrm{D} \mathrm{C}(\mathrm{r})=\mathrm{R}
$$

where $\mathrm{D}$ is the fractal dimension.

Since the ruler has a finite length, the details of the curve that are smaller than the ruler get skipped over and therefore the length we measure is normally less than the actual length of the curve. This can be seen in Figure 4 where, three rulers of different lengths are used to determine the length of the curve. The fractal dimension is estimated by measuring the length $L$ of the curve at various scale. Also it is true that as has been discussed in the use of ruler method starting point or origin position affects the count or number of boxes required, here too estimated value of $\mathrm{L}$ may vary depending on starting position. It is recommended that the same procedure be repeated at different starting position [4]. This method of determining the fractal dimension of a boundary or a curve is also referred as "structured walk". Longley and Batty [5] discuss number of variants of this basic procedure. Normant and Tricot [6] have described an alternative estimation algorithm, termed the 'constant deviation variable step (CDVS) method that emphasizes the local behavior of the curve [8-15].

\subsection{Self-similarity (Concept)}

The term self similarity came into existence about 40 years ago that too in a relation to Fractals and Fractal Geometry [4]. Fractal structures are said to be self-similar, when part of object looks like the whole object under fractal Dimension and Self-Similarity appropriate scaling i.e. the structure looks like a reduced copy of the full set ona different scale of magnification. The beauty of these clusters is that, each of these smaller clusters again is composed of still smaller ones, and those again of even smaller one. The second, third, and allthe following generations are essentially scaled down versions of the previousones. However this scaling can not be indefinitely extended, after certain stage the smaller pieces may not perfectly represent the original shape, this is the characteristic of natural fractals. In general this is termed as self-similarity or statistical self-similarity. Thus natural fractals exhibit self-similarity over a limited range and naturally occurring fractals usually exhibit statistical selfsimilarity [5] where as mathematical fractals exhibit self similarity at all length scales and thus are strictly self-similar.

Fractals are also strictly self-similar if it can be expressed as a union of sets. Geometric fractals may be composed of exact replicas of the whole object they are strictly self-similar [6].

After selecting the rectangular section of Figure 1 that exhibits the fractal properties of selfsimilarity, MATLAB was 
used to create a binarized version of the image (Figure 6) where any pixel above a certain threshold of color intensity was passed through as a one (true, shown in white) and the pixels below the threshold were passed through as a zero (false, shown in black). Passing this image through F. Moisy's box count package, boxcount. $\mathrm{m}$ [3].
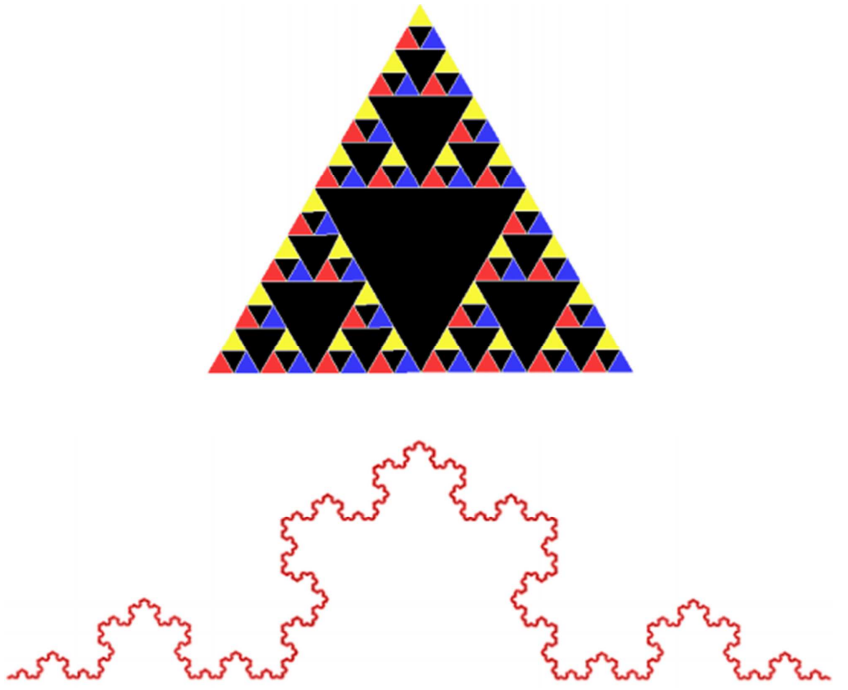

Figure 6. Sierpiniski triangles and Koch curve [8].

\section{Results}

By contrast, statistical fractals are self-affine, or statistically self-similar; they are composed of statistically equivalent replicas of the whole object. Examples of strictly self-similar fractals are Sierpiniski triangles, Koch flake etc as shown in Figure 6, the most fractal looking in nature do not display this precise from. The presence of self-similarity in the objects characterizes them as Fractal.

\section{References}

[1] Jiang B. and Yin J. (2014), Ht-index for quantifying the fractal or scaling structure of geographic features, Annals of the Association of American Geographers, 104 (3), 530-541.
[2] Jiang B. (2015), Geospatial analysis requires a different way of thinking: The problem of spatial heterogeneity, GeoJournal, 80 (1), 1-13.

[3] F. Moisy https://www.math.dartmouth.edu//archive/m53f09/public_htm 1/proj/Alexis_writeup.pdf.

[4] Fractal Dimension and Self-Similarity https://www.math.dartmouth.edu//archive/m53f09/public htm 1/proj/Alexis_writeup.pdf.

[5] Longley, P. A. and Batty, M. (1996), Spatial Analysis: Modelling in a GIS Environment, https://books.google.com.tr/books?isbn=0470236159.

[6] Normant, F. and Tricot, G., (1995) Fractals in Engineering https://www.google.com/search?q=Normant + and + Triart\&tbm $=$ isch \&source $=$ univ\&sa $=X \& v e d=2$ ahUKEwjgurqA5O hAhV SyqQKHeK7ChAQsAR6BAgJEAE\&biw $=1366 \& b i h=\overline{6}$.

[7] Yilmaz, L., "Maximum Entropy Theory by Using the Meandering Morphological Investigation-II", Journal of RMZ-Materials and Geoenvironment, 2007, CSA / ASCE, Printed also in CSA Illumina, CSA: Guide to Discovery, enews@csa.com.

[8] Sierpinski, S. (2002) https://www.mathsisfun.com/sierpinskitriangle.html https://wwwmathworld.wolfram.com/SierpinskiSieve.html.

[9] Kennedy, J. F., et al., Proc. Am. Soc. civ. Engrs, 97, 101-141 (1971).

[10] Hey, R. D., UK geol. Soc. Misc. Pap. 3, 42-56 (1974).

[11] Yalin, M. S., Mechanics of Sediment Transport (Pergamon, Oxford, 1972).

[12] Leopold, L. B., and Wolman, M. G., Bull. geol. Soc. Am., 71, 769-793 (1960).

[13] Leopold, L. B., Wolman, M. G., and Miller, J. P., Fluvial Processes in Geomorphology (Freeman, London, 1964).

[14] Shen, H. W., and Komura, S., Proc. Am. Soc. civ. Engrs, 94, 997-1015 (1968).

[15] Hey, R. D., and Thorne, C. R., Area, 7, 191-195 (1975). 